\title{
Posterior fracture-dislocation of the shoulder: an often unrecognized traumatic injury
}

\author{
Alfredo Figueiredo, André Pinto, Luís Corte-Real, Carlos Alegre, Rui Cabral, Fernando Fonseca \\ Department of Orthopaedic Surgery, Coimbra University Hospital, Portugal
}

Correspondence: Alfredo Figueiredo, Department of Orthopaedic Surgery, Coimbra University Hospital, Portugal, Email alfredojfigueiredo@gmail.com

Received: May 03, 2018 | Published: May 2I, 2018

Copyright@ 2018 Figueiredo et al. This is an open access article distributed under the terms of the Creative Commons Attribution License, which permits unrestricted use, distribution, and reproduction in any medium, provided the original author and source are credited.

\begin{abstract}
Posterior shoulder fracture-dislocation is a rare injury accounting for approximately $0.9 \%$ of shoulder fracture-dislocations. It is most often a result of highenergy trauma and a frequently overlooked diagnosis. The authors present the case of a patient admitted to the emergency department with a painful shoulder after a fall onto the outstretched arm. He was initially diagnosed a non-displaced fracture of proximal humerus based on radiographs of shoulder and upper arm. However, an asymmetric shoulder posterior outline lead to the request of a CT scan which then revealed a locked posterior fracture-dislocation of the shoulder. The patient was then taken to operating room to reduce the dislocation under general anesthesia and experienced a favorable evolution under conservative treatment. Shoulder functional outcomes are associated with early detection and treatment of these lesions. This article emphasizes the need for a high index of clinical suspicion of the injury in high-risk individuals making use of appropriate radiological investigation.
\end{abstract}

\section{Introduction}

Posterior shoulder dislocation is considered to be a rare injury accounting for only $2-5 \%$ of all shoulder dislocations. Even less frequent, posterior fracture-dislocation represents $0.9 \%$ of shoulder fracture-dislocations. ${ }^{1}$ Posterior shoulder dislocation is mainly caused by high-energy trauma such as motor vehicle accidents or other traumatic injuries in which patients suffer axial loading of the arm in an adducted, flexed and internally rotated position - an unstable combination for the shoulder joint. ${ }^{2}$ Impression fractures of humeral head articular surface, followed by humeral neck fractures and fractures of the lesser and greater tuberosity, are the more common associated fractures.

The majority of posterior shoulder dislocations occur in 35-55 year old men with high functional demand and $15 \%$ of these lesions are reported to be bilateral. ${ }^{3}$

Many radiographic signs of posterior dislocation on standard anteroposterior radiographs have already been described. ${ }^{4}$ However, these signs are unreliable and may not be present at the initial radiographic studies. Unless an axillary radiograph is requested, a single anteroposterior shoulder radiograph can be normal and lead to a misdiagnosis of the injury.

In fact, delays in diagnosis of posterior dislocations are common: it is estimated that up to $50 \%$ are missed when the patient first seeks medical consultation. ${ }^{5}$ The delay in diagnosis can lead to significant morbidity including chronic pain and stiffness as well as long-term functional disability.

There is no gold-standard treatment for posterior fracture-dislocation of the shoulder. Duration of dislocation, the number of bony fragments, patients' age and activity and the size of the humeral head defect are the main factors to take into consideration in the process of decisionmaking on patient's treatment. ${ }^{6,7}$

In this article, the authors present a clinical case that illustrates how the diagnosis of posterior-fracture dislocation of the shoulder can be misdiagnosed and how favorable the outcome can be if diagnosed early.

\section{Clinical case}

A 47 year old man was admitted to the emergency department of the institution with severe pain and functional limitation of his right shoulder. His past medical history was unremarkable and had no other complains. Hours before, he had suffered a fall onto an outstretched right arm. On physical examination, he presented with his upper limb showing a shoulder in internal rotation. He was moving his right hand spontaneously but the 'pain facies' raised the index of suspicion. He reported severe pain in palpation of his proximal upper arm. The shoulder active movement was markedly limited by the pain regardless the direction of movement. Passive joint movement was possible up to $45^{\circ}$ of flexion and abduction, but a mechanical block to external rotation was detected. The neurovascular status of the extremity was preserved.

A radiographic study of his right shoulder and upper arm was requested (Figures 1-4). It revealed an oblique fracture of proximal humerus without significant displacement. 


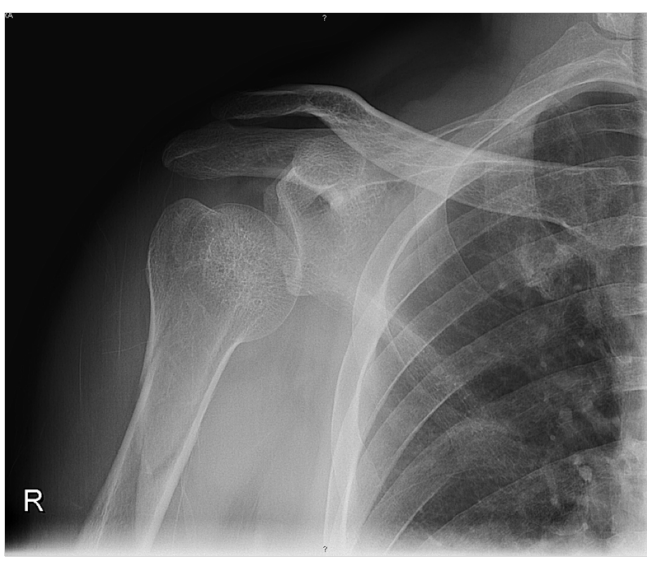

Figure I Shoulder X-ray (AP in plane of thorax) at the emergency department, arm in neutral rotation.

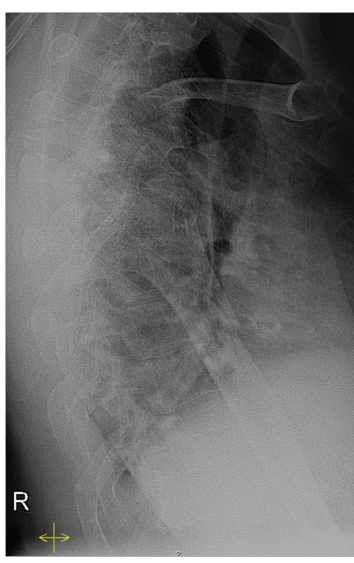

Figure 2 Shoulder $\mathrm{X}$-ray (right lateral) at the emergency department, arm in neutral rotation.

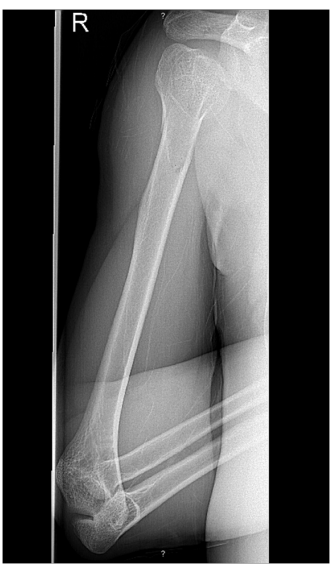

Figure 3 Upper Arm X-ray (AP in plane of thorax) at the emergency department.

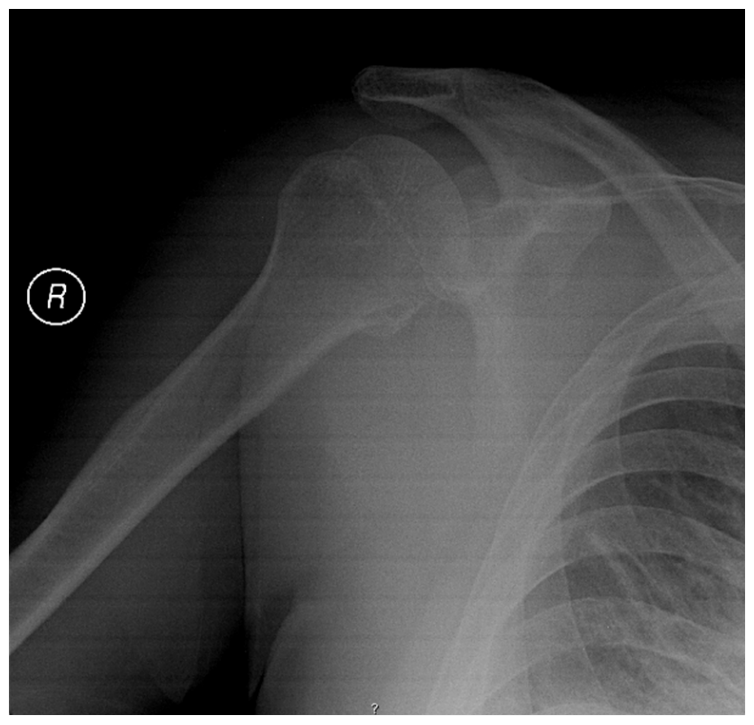

Figure 4 Shoulder X-ray (AP in plane of thorax) at the emergency department, arm in $20^{\circ}$ abduction.

The patient was submitted to conservative treatment. The intent was to immobilize the upper arm with a sling and providing additional support with a circumferential swath wrapping around the humerus and the chest.

It was only when starting the immobilization that a slight asymmetry of the shoulders was noticed. The painful shoulder had a posterior outline slightly more prominent. The procedure was discontinued and a CT scan of the shoulder was requested. The result was a locked posterior fracture-dislocation of the shoulder (Figures 5-8), with a compression fracture of the anteromedial portion of the humeral head involving less than $20 \%$ of the articular surface.

The patient was then submitted to a closed reduction of the shoulder under general anesthesia. It was a prompt reduction which benefited from muscle relaxation. With gentle but extensive maneuvers, it was possible to verify the stability of the reduction. A fluoroscopic evaluation of the reduction was made inside the operating room and the immobilization was then performed.

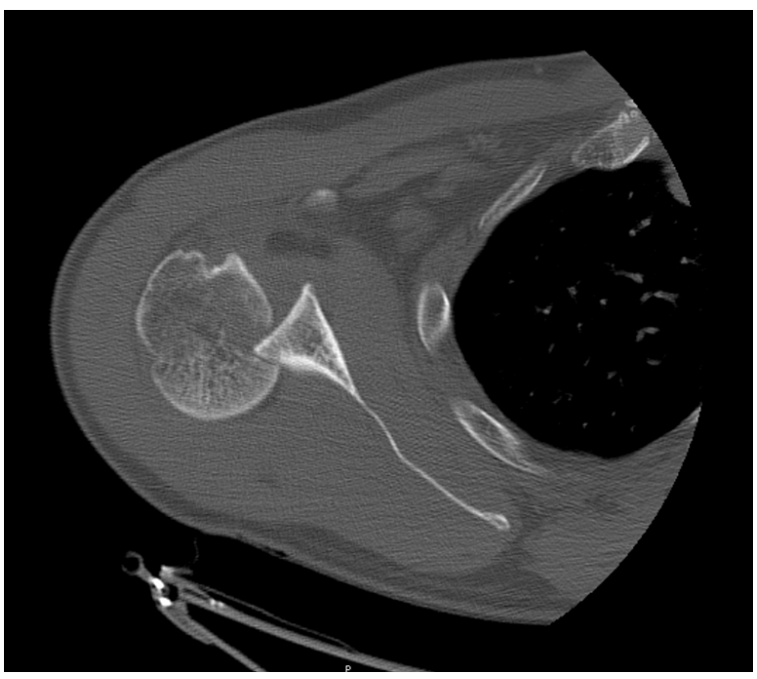

Figure 5 Shoulder CT image (transverse plane) at the emergency department.

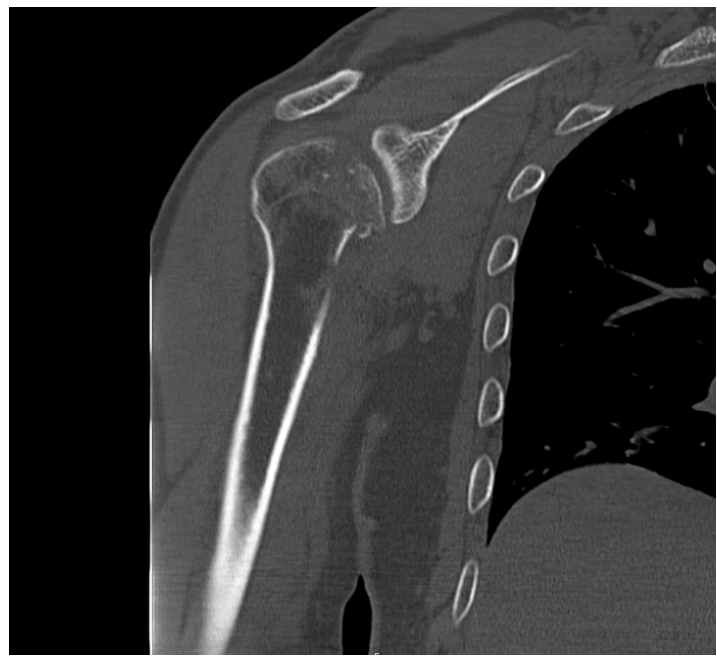

Figure 6 Shoulder CT image (coronal plane) at the emergency department. 


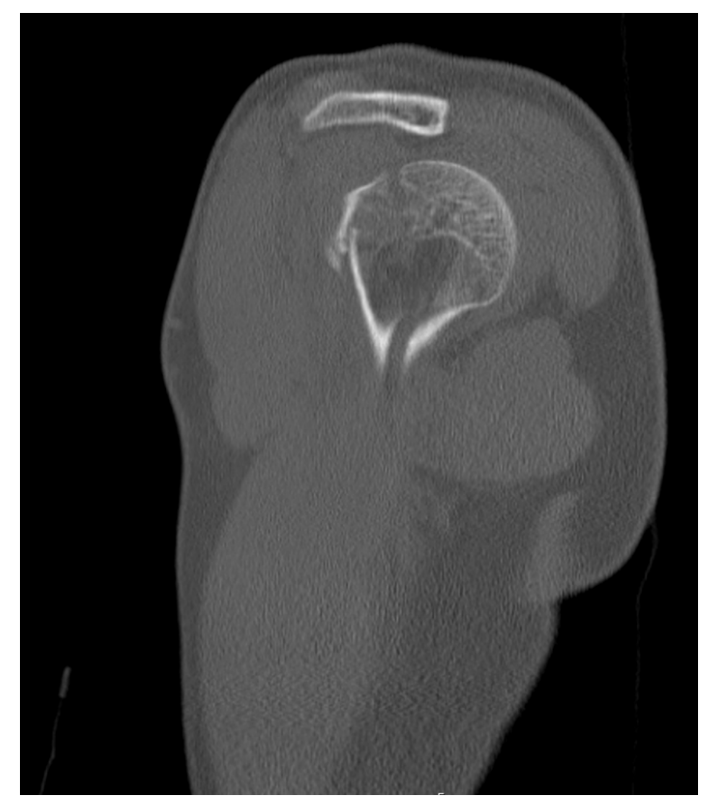

Figure 7 Shoulder CT image (sagittal plane) at the emergency department.

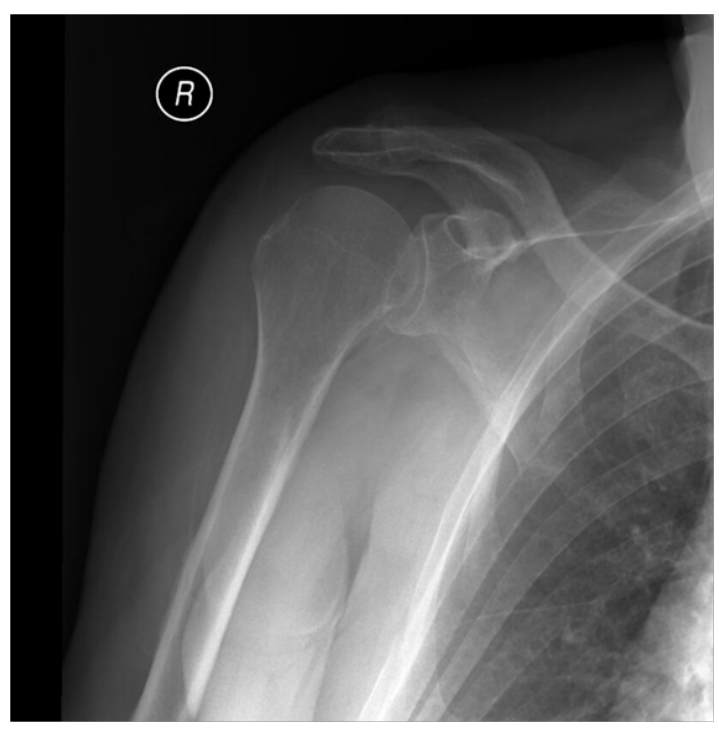

Figure 8 Shoulder X-ray (AP in plane of thorax) at 12 months of followup, arm in neutral rotation.

After three weeks, the patient started rehabilitation but used an arm sling for two more weeks. The clinical evaluation at 3 months revealed a patient doing his usual daily activities without significant symptoms and with a DASH score of 16.7. The patient was clinically and radiographically reevaluated at 12 months (Figure 8), reporting no complaints and with a DASH score of 8.4.

\section{Discussion}

The presented case illustrates how difficult it might be to diagnose a posterior fracture-dislocation of the shoulder when the orthopaedic surgeon is in the presence of a proximal fracture of the humerus. In fact, there is a high incidence of missed initial diagnosis ranging up to $79 \%$ in some studies. ${ }^{1}$ Common causes for delay include late presentation by the patient and inadequate radiographic investigation. Apical oblique, Velpeau or modified axial radiographs are the indicated views as they can be made with the arm in a sling.

In its normal position of function, the shoulder is protected from posterior dislocation by the stabilizing and buttressing action of the posterior aspect of the glenoid. The mechanical block to external rotation of the patient's right shoulder detected in the emergency room is a typical finding and is caused by engagement of the humeral head defect on the posterior aspect of the glenoid.

During posterior dislocation, an osteochondral impression fracture (also termed an encoche fracture or a reverse Hill-Sachs lesion) is produced as the anterior aspect of the humeral head impacts on the posterior aspect of the glenoid. ${ }^{9} \mathrm{CT}$ evaluation is mandatory to accurately detect and characterize the reverse Hill-Sachs lesion and complex fracture patterns if present.

The important distinction between fracture-dislocations, in which the humeral head is locked on the posterior aspect of the glenoid, and fracture-subluxations is unclear in most classification systems. ${ }^{7}$ This is why the authors have avoided the commonly used expression of "locked posterior fracture-dislocation of the shoulder".

The treatment of these injuries may be challenging as few surgeons encounter them with sufficient frequency in their clinical practice to be sure about the correct treatment strategy. The acute episode of posterior fracture-dislocation of the shoulder has two main treatment strategies: conservative and surgical. The surgical treatment is indicated in the rare cases when a closed reduction of the shoulder joint was not possible. A significant displacement of the humeral fracture is also an indication for surgical treatment. The fracture of our patient was a non-displaced fracture, for not having fulfilled the following criteria of fracture malalignment: $>20^{\circ}$ of anterior angulation, $>30^{\circ}$ varus/ valgus angulation and $<3 \mathrm{~cm}$ of shortening. The emergency surgical treatment of the humeral fracture can be performed with plate or by using Kirschner-wires as a provisional fixation. The compression fracture of the anteromedial portion of the humeral head is not usually managed in the emergency department. If superior to $20 \%$, it should be considered for operative treatment, either by arthroscopic or by open surgery. The patient defect of humeral head satisfied the conditions to be treated conservatively.

There is some evidence to suggest that posterior capsular tears will heal spontaneously following relocation of the shoulder. ${ }^{10}$ Outcomes have also been reported to be less satisfactory when closed reduction alone is not possible. ${ }^{3}$ These findings support the treatment that this patient was submitted to. The authors consider this is a wise option when the stability of the reduced shoulder joint is confirmed and also if the risk of post-traumatic shoulder osteoarthritis is accepted. It has been shown that post-traumatic degeneration of the glenohumeral joint is relatively uncommon after posterior dislocation, but when it occurs the severity of the degenerative osteoarthritis is usually worse than that following anterior dislocation. ${ }^{11}$

In what concerns to treatment results, no significant difference was found between the patients in which closed reduction was applied in the acutely treated posterior fracture-dislocation of shoulder and the cases that underwent anatomical reconstruction with a plate or Kirschner-wires. ${ }^{7}$ Furthermore, avascular necrosis of humeral head is a risk that must be taken into consideration if an open surgery is to be 
performed. ${ }^{8}$ However, chronic cases or acute cases with defects over $25 \%$ of the humeral head and complex fracture-dislocations demand open reduction and additional procedure to achieve stability. Shoulder arthroplasty, either hemi or total, is usually seen as a last option to treat chronic dislocations over 6 months, associated with defects over $45 \%$ or deformities of the humeral head. ${ }^{11}$

Persistent shoulder stiffness and functional incapacity after a simple dislocation are frequently associated with delay in diagnosis; other possible complications include deformity, osteoarthritis, or osteonecrosis of the humeral head. Joint stiffness may occur if a nonunion or malunion of the humeral head develops. The cause of stiffness is often obscure and is assumed to be due to periarticular fibrosis and capsular contracture. ${ }^{12}$

\section{Conclusion}

Posterior shoulder fracture-dislocation is a rare traumatic condition prone to be missed on initial presentation. Delays in diagnosis, errors in treatment, and protracted morbidity frequently occur. The patient described illustrates how easily this diagnosis can be overlooked, but on the other hand, how crucial it might be to shorten the diagnosis delay as much as possible. In fact, his satisfactory functional outcome largely benefited from prompt and accurate detection of the fracturedislocation. Neglected cases will frequently demand operative treatment and are associated with inferior functional outcome. Selection of the most appropriate treatment option can be issue of debate because of the rarity of these injuries. The key to diagnosis of posterior shoulder fracture-dislocations lies in maintaining a high index of clinical suspicion and performing appropriate radiographic investigations.

\section{References}

1. Kokkalis ZT, Iliopoulos ID, Antoniou G, et al. Posterior shoulder fracturedislocation: an update with treatment algorithm. Eur J Orthop Surg Traumatol. 2017;27(3):285-294.
2. Sheehan SE, Gaviola G, Gordon R, et al. Traumatic shoulder injuries: a force mechanism analysis - glenohumeral dislocation and instability. AJR Am J Roentgenol. 2013;201(2):378-393.

3. Checchia SL, Santos PD, Miyazaki AN. Surgical treatment of acute and chronic posterior fracture-dislocation of the shoulder. J Shoulder Elbow Surg. 1998;7(1):53-65.

4. Arndt JH, Sears AD. Posterior dislocation of the shoulder. Am J Roentgenol Radium Ther Nucl Med. 1965;94:639-645.

5. Hawkins RJ. Unrecognized dislocations of the shoulder. Instr Course Lect. 1985;34:258-263.

6. Kowalsky MS, Levine WN. Traumatic posterior glenohumeral dislocation: classification, pathoanatomy, diagnosis, and treatment. Orthop Clin N Am. 2008;39(4):519-533.

7. Basal O, Dincer R, Turk B. Locked posterior dislocation of the shoulder - a systematic review. EFORT Open Rev. 2018;3:15-20.

8. Robinson CM, Akhtar A, Mitchell M, et al. Complex posterior fracturedislocation of the shoulder. Epidemiology, injury patterns, and results of operative treatment. J Bone Joint Surg Am . 2007;89(7):1454-1466.

9. Edelson G, Kelly I, Vigder F, et al. A three-dimensional classification for fractures of the proximal humerus. J Bone Joint Surg Br. 2004;86(3):413425 .

10. Gerber C. Chronic, locked anterior and posterior dislocations. In: Complex and revision problems in shoulder surgery. 1997:99-116.

11. Samilson RL, Prieto V. Dislocation arthropathy of the shoulder. $J$ Bone Joint Surg Am. 1983;65:456-460.

12. Robinson CM, Aderinto J. Posterior shoulder dislocations and fracturedislocations. J Bone Joint Surg Am. 2005;87(3):639-650. 